Man and Nature

L'homme et la nature

\title{
Les fondements du savoir dans la pensée moraliste des Lumières
}

\section{Henri Mydlarski}

Volume 7, 1988

URI : https://id.erudit.org/iderudit/1011931ar

DOI : https://doi.org/10.7202/1011931ar

Aller au sommaire du numéro

Éditeur(s)

Canadian Society for Eighteenth-Century Studies / Société canadienne d'étude du dix-huitième siècle

ISSN

0824-3298 (imprimé)

1927-8810 (numérique)

Découvrir la revue

Citer cet article

Mydlarski, H. (1988). Les fondements du savoir dans la pensée moraliste des Lumières. Man and Nature / L'homme et la nature, 7, 141-148.

https://doi.org/10.7202/1011931ar

Copyright (C) Canadian Society for Eighteenth-Century Studies / Sociéte canadienne d'étude du dix-huitième siècle, 1988
Ce document est protégé par la loi sur le droit d'auteur. L'utilisation des services d'Érudit (y compris la reproduction) est assujettie à sa politique d'utilisation que vous pouvez consulter en ligne.

https://apropos.erudit.org/fr/usagers/politique-dutilisation/ 


\section{Les fondements du savoir dans la pensée moraliste des Lumières}

Il n'est guère d'écriture moraliste, de quelque période que ce soit, qui ne réfléchisse, au moins en filigrane, une théorie de la connaissance. Il n'en est pas qui, en matière de certitude, ne finisse par se dérober aux primats de l'objectivité. Tous les moralistes français le savent. Et parce qu'ils en conviennent volontiers, lors même qu'ils se retranchent derrière un rationalisme de l'absolu, ils conjuguent aux injonctions de la modalité démonstrative les inflexions des arcanes oniriques et les retombées de l'histoire. Car il n'y a pas de connaissance, pour eux, ni d'écriture qui se refuse à puiser dans les incantations du 'moi' et les moments de l'humanité. Leçon que ferait peut-être bien de ne pas dénoncer une pensée qui aime aujourd'hui à se proclamer scientifiquement une par sa définition du savoir et par son ancrage dans une même approche de la vérité. 'Evidence, logique, déduction, ne sont que des fantômes vides. Une vérité "devient" évidente, logique, déduite, mais après l'acte créateur,' ne cesse de répéter par exemple Saint-Exupéry. ${ }^{1}$

Selon les moralistes des Lumières comme pour ceux du Grand Siècle, il se découvre à l'homme deux grands espaces du savoir: l'espace de la facticité et l'espace de la signification, c'est-à-dire le champ d'une praxis scientifique qui instituerait la connaissance phénoménale et le champ d'une doxa qui investirait le monde de sa finalité. Mais si les uns et les autres paraissent se rencontrer aux frontières d'une topographie épistémologique commune, ils divergent, en revanche, par les perspectives qui apriorisent les approches respectives de leur mode de connaissance. Chez les moralistes 'classiques,' la doxa se reçoit des mains de Dieu par un acte de sa miséricorde et par la médiation apostolique de l'Eglise. Ainsi Pascal, parce qu'on ne saurait comprendre les parties qu'en fonction du tout (en même temps d'ailleurs qu'on ne saurait comprenäre le tout qu'en fonction des parties), 'crucifie-t-il,' jusque dans les aires d'investigation les plus radicalement profanes, toutes les prétentions scientifiques à la totalisation ou à la déconstruction. D'où l'hérésie de la libido sciendi, hérésie si condamnable à ses yeux que son neveu Florin Périer, en une préface aux Traités de l'équilibre 
des liqueurs et de la pesanteur de la masse de l'air, écrits scientifiques s'il en est, se fait un devoir, pour le moins inattendu dans de pareils ouvrages, de rappeler que Pascal sentait 'la vanité et le néant de toutes ces connaissances et [qu']il en avait conçu un tel dégoût qu'il avait peine à souffrir que des personnes d'esprit s'y occupassent ou en parlassent sérieusement. ${ }^{2}$ Entendons bien: il s'agit beaucoup moins ici de sanctionner le faire-valoir de 'l'unique nécessaire' dont parle Saint Luc, de sacrifier à l'espoir d'une vie béatifique les acquis d'un siècle avancé, que de réduire à l'inanité les revendications de la science; il s'agit de mettre au pilori toute doctrine rationaliste ou (avant son heure) positiviste qui prétendrait donner accès à la nature de l'homme et des choses en soi. Davantage: Pascal va jusqu'à nier que puisse se dessiner dans 'l'ordre des éléments' ${ }^{3}$ - l'expression est de lui - une écologie de rapports fondée en système et qui fonderait à son tour en cohérence le champ de la recherche physique et scientifique. ${ }^{4}$ Que le monde se maintienne, non, il ne le doit pas à d'hypothétiques déterminations du Logos, à de prétendues lois cosmiques et universelles, il en est redevable exclusivement à la bonté de Dieu. Car n'est pas seulement impuissante la raison de l'homme face à l'univers, encore se doitelle de constater (ce qu'elle est quand même, malgré sa faiblesse, en mesure de constater) qu'il n'est rien dans ce même univers qui ne soit totalité brisée, mutilée et polypeuse.

Sans Jésus-Christ, écrit Pascal dans un de ses fragments les plus achevés et les plus longs, le monde ne subsisterait pas; car il faudrait ou qu'il fût détruit, ou qu'il fût comme un enfer. ${ }^{4}$

Et ailleurs, pour dire que chaque chose sur terre relève d'une disparité et d'une irréductibilité fondamentales; que la moindre production sensible opère en vase clos: 'La nature a mis toutes ses vérités en soi-même. Notre art les renferme les unes dans les autres, mais cela n'est pas naturel. Chacune tient sa place. ${ }^{5} \mathrm{Il}$ y a plus. L'écriture même des Pensées restitue moins l'état d'une ouvre inachevée qu'elle ne porte témoignage, par sa matérialité diffuse et son déchirement interne, de l'imposture qu'il y a à tenir tout discours conjonctif (scientifique ou autre) sur le modèle de 'la méthode,' comme le rêvait Descartes, ou sur le principe de 'la chaîne dorée,' comme y invitait le pourtant sceptique La Mothe le Vayer. ${ }^{6}$ En un mot, il n'y a pas de tessiture de la connaissance parce qu'il n'y a pas de tessiture du monde. Pour connaître la raison de toutes choses, il faut connaître Jésus-Christ, qui est 'l'objet de tout, et le centre où tout tend. ${ }^{7}$ Et par cette vision universelle s'explique encore que ne soit pas même venue à Pascal - ni, au demeurant, aux autres moralistes du XVIIe siècle - l'idée d'une pos- 
sible science des phénomènes sociaux. Que ceux-ci aient planté un important jalon en édifiant le primat de l'expérimentation en physique; qu'ils aient fait progresser nos connaissances dans d'autres domaines, notamment en 'psychologie,' non, certes, il n'y a pas là l'ombre d'un doute. Mais il n'est pas moins indubitable qu'ils posent, en amont et en aval de leur réflexion, des impératifs contra-scientifiques à l'étage de la recherche pure et qu'ils y dressent des apories accablantes, singulièrement hostiles à l'approfondissement des vérités élémentaires et à de nouvelles découvertes. A ce train, ils s'inscrivent même en faux contre l'esprit de leur temps. ${ }^{8}$

Chez les moralistes des Lumières, en revanche, la doxa qui établirait la vérité et ouvrirait la voie-norme du savoir s'érige sur un dynamisme de lois qui auraient de toujours régi l'univers. Ils substituent en effet à la disjonction de deux ordres axiologiques (institués respectivement, rappelons-le, en raison divine et en raison atrophiée) une nodale du Logos qui fonderait en harmonie la construction du monde et de la créature. Ils vont même jusqu'à faire retentir chez l'homme des pulsions qui le porteraient à vibrer à l'unisson avec l'univers. Mieux: ils s'essaient, à l'occasion, à en reproduire la tonalité et l'amplitude en une sorte de mise en abyme affective. Car ils ne cessent pas seulement de répéter que la nature ne saurait se contredire, énonçant ainsi un critère premier de la connaissance rationaliste, ils avancent aussi, tel Vauvenargues, le principe d'une adéquation entre le sentiment profane et la téléologie:

[Une grande âme] incorpore à soi toutes les choses de la terre; elle tient à tout; tout la touche; rien ne lui est étranger: ni la différence infinie des mours, ni celle des conditions, ni celle des pays, ni la distance des temps, ne l'empêchent de rapprocher toutes les choses humaines, de s'unir d'intérêt à tout. ${ }^{9}$

En d'autres termes, les moralistes des Lumières ne font pas qu'évacuer de l'infini l'effroi qui hantait Pascal, ils y versent, pour mieux investir la réflexion de son droit de cité au royaume du savoir, la richesse d'une conscience qui remonterait aux temps lointains et mystérieux où se seraient constitués le monde, les hommes et les hérédités. Il y a quelques mois, lors d'une conférence à l'université de Calgary le célèbre linguiste Thomas Sebeok déclarait:

When language first developed two million years ago it wasn't used for communication. It arose instead because our ancestor homo habilus had developed the capacity to create in its mind possible worlds. ${ }^{10}$

Les linguistes d'aujourd'hui commenceraient-ils à rebrousser chemin et à se prévaloir, sans s'en rendre toujours bien compte, de la leçon 
des linguistes d'hier que furent aussi les moralistes du XVIIIe sièlcle? J'ai bien dit: commenceraient. Car ceux-ci étayent les fondements du savoir d'une approche autrement plus radicale. Il abordent en effet le problème de la connaissance à partir du concept, pour employer une expression (peut-être trop) moderne, d'un 'clonage en réduction' du monde par l'individu à l'instant même de la Création, si Création il $\mathrm{y}$ a eu. Ils s'engagent à montrer, chacun selon son inclination et sa démarche personnelles, que notre 'moi' ou la raison ou encore les deux ensemble par manière de symbiose, ne sont jamais, après tout, que les expressions irisées du Logos et, par voie d'identité, d'une même intelligence de la réalité. Dans la consubstantialité de l'homme avec le monde s'estompe à leurs yeux, pour la plus grande gloire de l'homme, l'énigme du monde; se fait jour, dans le sens de l'ici-bas, le sens de l'être:

il n'est rien dans la nature, écrit encore Vauvenargues, qui ne soit une image de la vie humaine, parce que la vie humaine est elle-même une image de toutes choses, et que tout l'univers est gouverné par les mêmes lois. ${ }^{11}$

Mieux encore: le plus rationaliste des écrivains moralistes des Lumières, François Vincent Toussaint, se voit amené à conclure avec un malin plaisir que l'intellectualité trouve sa source et sa vigueur dans la physiologie du phénomène passionnel.

Otez à un homme toutes ses passions, écrit-il, le voilà devenu un pur automate, et même un automate sans jeu: car ce sont les passions qui mettent en action, non seulement la partie animale mais souvent même aussi la partie spirituelle. A mesure donc que vous lui ôtrez [sic] une de ses passions, vous l'approcherez d'un degré, de l'inertie; mais réglez-les ou les modérez toutes; et voilà aussi-tôt ses facultés, tant sensitives que spirituelles, qui agissent de concert; et tous ses talens et toutes ses vertus qui se développent et s'étalent. ${ }^{12}$

De fait, pour l'auteur des Mours, le vérité et la connaissance tout ensemble participent d'une telle réception/adhésion subjective du 'je' à l'ordre des choses que ne se pose même pas pour lui le problème de l'âme et de la mort. ${ }^{13} C^{\prime}$ 'est dire que les écrivains qui nous occupent ici ont beau, selon les besoins de leur thèse, se réclamer de Descartes, ils s'en affranchissent pour ruiner son dualisme. Assurément, loin de désavouer les passions comme des maladies de l'âme, le philosophe les avait mises au service du corps et de la pensée, préférant 'utilise[r] au profit du progrès moral la richesse concrète de la vie affective. ${ }^{\prime 14}$ Mais il avait également, dans le cogito, saisi l'âme comme raison pure el rappelé, dans son traité des passions, qu'elle ne pouvait être com- 
promise par son union avec le corps, par son adhérence à l'écologie animale. ${ }^{15}$ Rien de tel chez nos moralistes. Pour eux la destinée de l'homme n'est pas plus surnaturelle que les lois mêmes de la nature dont elle émane et qu'elle reflète dans son rayonnement. Ils ne voient ni motif ni intérêt à se persuader, comme se le persuadait Pascal qui se rencontrait ici - malgré lui - avec Descartes,

[qu']il est impossible que la partie qui raisonne en nous soit autre que spirituelle et [que] quand on prétendrait que nous serions simplement corporels cela nous exclurait bien davantage de la connaissance des choses. ${ }^{16}$

Paradoxalement, ceux qui s'égarent sont, à leurs yeux, ceux-là mêmes qui s'entêtent à compartimenter le monde; à diviser la finalité indivisible de l'absolu, et à parler, comme si les unes ne formaient pas avec les autres un tout homogène, des choses corporelles corporellement et des choses spirituelles spirituellement. ${ }^{17}$ Est portée au compte de l'imposture ou de l'aliénation, chez Duclos, Vauvenargues, Toussaint et, plus tard, chez Sénac de Meilhan et Chamfort, toute prétendue dichotomie qui, instituant le monde et la connaissance en vanité ('vanité,' à l'origine, signifie proprement: caractère de ce qui est vide), hypostasierait en revanche le souvenir d'un être un, total et divorcé du temps et de l'espace.

Le caractère change, écrit par exemple Sénac de Meilhan pour attester qu'il n'est rien qui ne soit en devenir, les situations de la fortune varient, les affaires se multiplient, l'humeur s'altère, les goûts s'affoiblissent, de nouveaux attachements remplissent le coeur de l'homme, il devient époux et père, de nouveaux rapports s'établissent entre lui et les autres, et l'on veut qu'il y ait un attachement qui survive à toutes ces vicissitudes, qui ne perde rien de son action, de son charme, et qui remplisse également le cour? ${ }^{18}$

A la limite, la conception moraliste du savoir s'ouvre sur une dynamique moniste de l'ambivalence unifiée. Elle inscrit au coeur même des exigences physico-mathématiques que commande aujourd'hui le champ sémantique du savant, l'irrationalité du coeur, les incursions de l'affectivité: il n'est pas de discours en science qui puisse se tenir sur le mode de l'excommunication de l'être; il n'est pas de méthodologie, y compris logico-phénoménale, qui ne soit en relation de sous-main avec le sentiment. Au Ciel comme sur Terre, l'onirique et la matière se mêlent pour s'unir, au sens premier du mot; pour créer esprit et vie.

Un problème pratique pourtant se pose. Quand même la connaissance reposerait sur un système d'explication et un contingent humain 
d'aptitudes - contrairement à Pascal qui croyait que ce qui passe la géométrie nous surpasse - quant même serait atteinte et dominée la finalité du monde, il reste que les sciences dites 'exactes' ou 'dures' conduisent irréductiblement à une véritable taxonomie de la méthode et du savoir. La vie humaine a beau être une image en réduction de l'univers et de ses lois, la pensée qui se propose d'intégrer la complexité et l'abondance du monde se voit astreinte, au seuil de toute enquête sur le phénoménal, à une parcellisation du savoir, à une régionalisation de la recherche. De la connaissance dynamique et globale qui donne aux choses leur raison d'être, à ces choses elles-mêmes, dans leur foisonnante latitude, pèse, du poids de l'interdit, l'hypothèque d'une incommensurable instance plurielle. Et cette pluralité, comme cette incommensurabilité, n'en finit pas de finir. Elle ne cesse pas de repousser les limites de la science en un dehors toujours plus lointain, au grand dam de l'intelligence analytique et dissociante. Les moralistes des Lumières savent très bien que tel est le lot de la science. Ils n'ignorent pas qu'elle est à jamais contrainte, sui generis, à explorer sans relâche ni répit des régions nouvelles et de nouvelles possibilités. Pourtant, ils ont espoir qu'elle aidera à restituer un jour à l'homme sa dignité perdue, sa grandeur primitive. Elle, dont les réseaux semblent si difficilement s'articuler avec leur vision de la réalité et sur laquelle ils ne peuvent s'empêcher quelquefois d'ironiser! Mais les moralistes ont également conscience qu'elle est appelée, aujourd'hui plus que jamais, à progresser parallèlement à l'évolution des mentalités. Aussi se feront-ils un devoir de revendiquer, à une époque qui paraît du reste s'ouvrir chaque heure davantage à elle, son utilité pratique et sa valeur morale. Par là ils penseront ruiner l'arbitraire des philosophies et des théologies précédentes, et élucider après des siècles d'obscurantisme, à travers une consonance du subjectif avec l'objectif, les déterminations inhérentes à la nature des choses et de la condition humaine.

Que conclure? Je me contenterai de faire une seule remarque. Il est de bonne pratique, dans notre société, de ne point assimiler vérité et savoir. L'on se plaît, parce que, il faut bien l'avouer, cela apaise souvent, à disjoindre science et sens; à prétendre que la production des vérités transcendantes qui naissent du sentiment intérieur ne saurait se justifier - et encore moins se définir - en corrélation avec la représentation scientifique. Parfois même nos savants, par crainte peutêtre de lèse-scientificité, se font fort de démentir que puisse tant soit peu loger, à la source de leur 'impartialité,' une notion d'homme et d'univers soit individuelle soit collective qui imprimerait à leurs efforts une direction fixée d'avance. Peut-être en est-il ainsi? Mais ce n'est certainement pas ce que nous apprend l'exemple des moralistes des Lumières face à la leçon des moralistes du Grand Siècle. Les uns et les 
autres prouvent historiquement de même que spirituellement qu'il n'est pas de savoir (ou de manque de savoir) qui ne plonge des racines dans une attitude, dans une idée aprioriste de la vérité; que l'acte de connaître est à l'origine un acte d'espoir et, à sa façon, de foi au monde et en la créature. Peut-être même prouvent-ils, comme le proclame Saint-Exupéry que j'ai cité au début de ces pages, que 'ce qui sera créé, ce qui naîtra sera logique. Et non: ce qui est logique naîtra. ${ }^{19}$

\section{HENRI MYDLARSKI}

Université de Calgary

\section{Notes}

1 Antoine de Saint-Exupéry, Carnets (Paris: Gallimard, 1975), p. 234.

2 Blaise Pascal, Oeuvres complètes (Paris: Seuil, 1963), p. 233.

3 Pascal, ibid., p. 558.

4 Pascal, ibid., p. 558.

5 Pascal, ibid., p. 590.

6 François de La Mothe le Vayer, Oeuvres, Tome II, Partie I (Dresde: Michel Groel, 1756), p. 356.

7 Voir Pascal, op. cit., p. 558.

8 Sur la question de la conception pascalienne du discours, Louis Marin écrit: 'la pratique pascalienne du discours, en réalisant dans et par son écriture propre - fragmentation, interruption, digression - la neutralisation du discours de savoir, de sa théorie logico-grammaticale et de ses appuis métaphysiques, opérait, dans l'ordre du langage et du discours, la critique la plus radicale qui était possible de l'idéologie qui en était à la fois le produit et le résultat: celle de la représentation' (souligné dans le texte), La Critique du discours - sur la 'Logique de Port-Royal' et les 'Pensées' de Pascal (Paris: Les Editions de Minuit, 1975), p. 418.

9 Vauvenargues, Oeuvres et Oeuvres posthumes et auvres inédites, éd. D.-L. Gilbert (Paris: Furne et Cie., 1857) II, 29.

10 Thomas Sebeok, 'Language not for Communicating,' The University of Calgary Gazette, Vol. 16, No. 5 (14 mai 1986), 3.

11 Vauvenargues, op. cit. II, 460.

12 [Toussaint], Eclaircissement sur les Mours (Amsterdam: Marc Michel Rey, 1762), p. 225. 
13 Voir notre 'Regard sur le moraliste français,' Tijdschrift voor de Studie van de Verlichting en van haet vrije Denken, 13, Nos. 2-3 (1985), 104.

14 Geneviève Rodis-Lewis, 'Introduction' in Descartes, Les passions de l'âme (Paris: Librairie Philosophique J. Vrin, 1966), p. 14.

15 Descartes écrit par exemple: 'l'âme ne s'absente lors qu'on meurt, qu'à cause que cette chaleur [la chaleur naturelle qui meut les corps 'inanimés'] cesse, et que les organes qui servent à mouvoir le corps se corrompent,' op. cit., p. 68 . Et ailleurs: 'à cause que nous ne concevons point que le corps pense en aucune façon, nous avons raison de croire que toutes les sortes de pensées qui sont en nous appartiennent à l'âme; Et à cause que nous ne doutons point qu'il n'y ait des corps inanimés, qui se peuvent mouvoir en autant ou plus de diverses façons que les nostres ... nous devons croire que toute la chaleur et tous les mouvemens qui sont en nous, en tant qu'ils ne dépendent point de la pensée, n'appartienent qu'au corps' (pp. 67-68).

16 Pascal, op. cit., p. 527.

17 Cf. Pascal, ibid., p. 527.

18 Sénac de Meilhan, Considérations sur l'esprit et les mœurs, éd. Fernand Caussy (Paris: E. Sansot et Cie, Igos), pp. 132-33.

19 Saint-Exupéry, op. cit., p. 154. 